\title{
Optimal Cluster Head Selection Schemes for Hierarchical OFDMA Based Video Sensor Networks
}

\author{
Rituraj \\ Department of Electrical Engineering \\ Indian Institute of Technology Kanpur \\ Kanpur, India 208016 \\ Email: rituraj@iitk.ac.in
}

\author{
Aditya K. Jagannatham \\ Department of Electrical Engineering \\ Indian Institute of Technology Kanpur \\ Kanpur, India 208016 \\ Email:adityaj@iitk.ac.in
}

\begin{abstract}
In this paper we present a novel framework for cluster head $(\mathrm{CH})$ selection and sub-carrier allocation towards intra-cluster communication in an Orthogonal Frequency Division Multiple Access (OFDMA) based Wireless Sensor Network (WSN). The proposed framework maximizes the overall performance of the WSN in terms of the throughput, video quality and network life. The OFDMA PHY optimization criterion for rate maximization is formulated as a bi-level optimization problem for CH node selection followed by optimal OFDMA sub-carrier allocation. It is then demonstrated that the above optimal resource allocation problem can be reduced to the solution of a weighted bipartite graph matching problem and is solved employing three different schemes, namely the popular Hungarian algorithm, game theory based multi-item auction and the Gale-Shapley stable matching scheme. Based on the above formulation, we also derive an optimal scheme to minimize the energy consumption in the WSN. Further, the performance of the proposed schemes is demonstrated through simulations in the context of scalable video transmission in a video sensor network. Results illustrate that these schemes significantly outperform suboptimal WSN cluster head selection schemes in terms of data rate and video quality.
\end{abstract}

\section{INTRODUCTION}

Wireless Sensor Networks (WSN) are rapidly gaining popularity in various critical areas such as video surveillance, monitoring, defense and security applications [1, chap. 2]. A WSN consists of a large number of sensor nodes measuring parameters such as temperature, pressure etc. in a local area and interconnected through wireless links with the aim of relaying the sensor measurements to a central data sink. The wireless sensor nodes are typically battery powered and have limited energy, necessitating the development of optimal transmission schemes to maximize the WSN performance. Further, uneven load on the sensor nodes can potentially result in some nodes depleting their batteries earlier and thus disrupting the network connectivity, leading to network life reduction.

Towards this end several architectures and routing protocols have been proposed for an efficient and reliable communication in WSNs. Although multi-hop routing minimizes the total energy consumption, thus increasing the network life, a serious drawback of multi-hop routing is the traffic

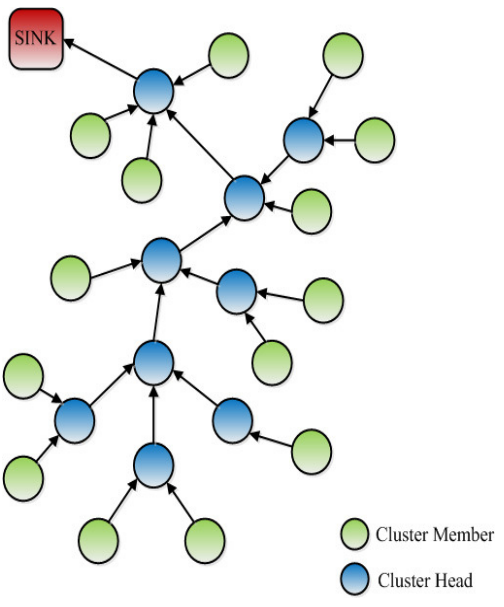

Figure 1: Hierarchical Cluster Based WSN

congestion in the nodes closer to the base station leading to faster energy depletion of these nodes. In this context, the class of hierarchical routing protocols have attracted significant research interest due their several advantages such as scalability, interference minimization and uniform load distribution arising as a result of the hierarchical WSN architecture [1. p. 148]. The hierarchical WSN architecture, shown in Fig1 is based on grouping the sensor nodes into clusters followed subsequently by choosing a cluster head $(\mathrm{CH})$ in each cluster. The $\mathrm{CH}$ then performs the task of intra-cluster data aggregation, followed by local sensor fusion and transmission of the relevant information to the data sink through other intermediate CHs. Since communication with the distant base station is significantly energy consuming, one can significantly decrease energy consumption by restricting a dominant fraction of the $\mathrm{CH}$ communication to intra-cluster i.e. with the nodes in the cluster followed by intelligent local sensor fusion. Thus, in order to maximize the overall WSN performance, one has to optimize the intra-cluster communication efficiency. Further, it can be noted that since the $\mathrm{CH}$ job is more energy demanding, it is mandatory to keep rotating the $\mathrm{CH}$ assignment 
among the nodes of the cluster which naturally leads to uniform energy consumption over the WSN lifetime. Our work achieves efficient intra-cluster communication through a two level optimization: optimal CH selection followed by OFDMA sub-carrier allocation among the nodes in the cluster with the aim of WSN throughput and video quality maximization. Also, the statistically symmetric random fading nature of the wireless channel leads to a uniform $\mathrm{CH}$ load distribution in the proposed scheme. Hence, the proposed approach enhances the lifetime of the WSN compared to the conventional approaches.

Several clustering algorithms have been proposed in the literature [1], [2]. Base-Station Controlled Dynamic Clustering Protocol (BCDCP) [3] is a scheme for stable and uniform Clustering, which is done during network configuration by the base station. For adaptive and distributed clustering, several techniques such as HEED, LEACH or MRECA have been proposed. Typical distributed clustering algorithms comprise of a two stage procedure involving $\mathrm{CH}$ node assignment followed by cluster formation. The serious drawback of these approaches is that $\mathrm{CH}$ selection is done solely on the basis of residual node energy to equalize the energy consumption among all the nodes. Hence, generally the performance of the WSN is compromised to ensure even load distribution.

Further, all of the above mentioned clustering protocols are described for a fixed TDMA scheduling based WSNs. However, our work shows that employing an OFMDA based scheme provides greater benefits due to the frequency diversity inherent in the system. In the OFDMA based framework we employ one of the above mentioned popular algorithms for clustering followed by intra-cluster optimization, comprising of a short setup phase followed by the steady state phase. The authors in [4] propose an algorithm based on the water filling method to optimally allocate subcarriers in OFDMA framework such that the sum data rate is maximized for a given total transmit power constraint. In a WSN, the maximum transmit power of each node is limited by the battery status. These additional constraints make the existing resource allocation schemes for an OFDMA system inapplicable for WSNs. So for sensor-subcarrier allocation we present a novel time-frequency resource allocation as shown in Fig.2. In the setup phase, the best node is selected as $\mathrm{CH}$ which then optimally assigns the subcarriers to the remaining nodes. In dynamic scenarios, reclustering can also be done after a suitable number of intracluster optimization cycles. For simplicity we assume a single subcarrier per sensor node and equal transmission power for all nodes. However, the proposed schemes are general and can be readily applied to unequal transmission power scenarios Further, if the difference in the distances of the nodes in the cluster is negligible, our optimization scheme not only achieves optimum sum data rate but also harnesses the channel randomness to achieve uniform energy consumption among the nodes in the cluster.

The rest of this paper is organized as follows. In section II we describe the system and video quality models employed in this paper. We consider scalable video coding for video transmission because of its adaptive nature [5, chap. 11].

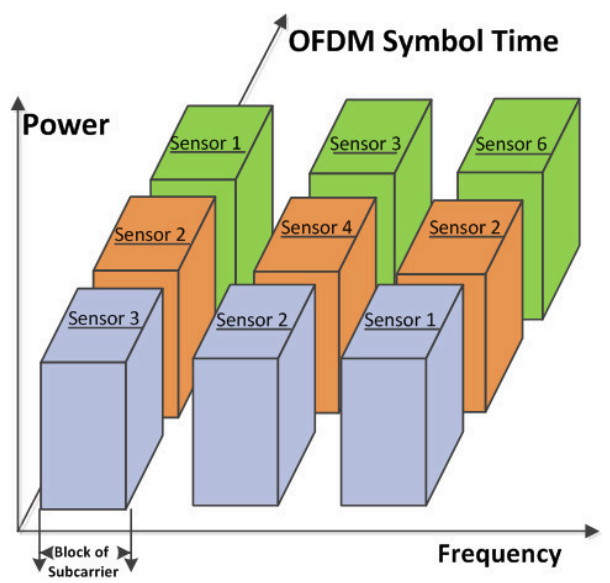

Figure 2: WSN OFDMA Subcarrier Allocation

Authors in [6], [7], [8] present a very good survey on wireless multimedia sensor networks. In section III we describe the stable matching, multi-item auction based bipartite matching and Hungarian algorithm for $\mathrm{CH}$ selection and OFDMA subcarrier allocation. We also briefly describe how these algorithms can be employed to minimize energy consumption or maximize sum video quality in section III. The performance comparisons of the proposed schemes and simulation results are given in section IV. Finally we conclude the paper in section V.

\section{SYSTEM MODEL}

Consider a WSN cluster consisting of $N$ sensor nodes and $(N-1)$ OFDMA subcarriers which can be allocated to individual sensor nodes for intra-cluster communication. The $\mathrm{CH}$ assignment problem is to choose the $\mathrm{CH}$ and allocate the subcarriers from the $N$ ! diverse channel allocations in total for a cluster containing $N$ nodes resulting from $N$ possible choices for the $\mathrm{CH}$ followed by $(N-1)$ ! possible subcarrier allocations. Let the fading channel gain between the $i^{\text {th }}$ node over the $j^{\text {th }}$ subcarrier for choice $\xi$ of the cluster head be denoted by Rayleigh coefficient $h_{i, j}(\xi)$. Thus, the size of Channel gain matrix $\mathbf{G}(\xi)$ is $(N-1)^{2}$ corresponding to $N-1$ rows, one for each node other than the choice of $\mathrm{CH} \xi$. Since a node can communicate with the $\mathrm{CH}$ on any of the $(N-1)$ subcarriers, there are $(N-1)$ columns. The maximum achievable data rate $R_{i, j}(\xi)$ for reliable communication over bandwidth $B$ and transmit power $P$ is given as,

$$
R_{i, j}(\xi)=B \log _{2}\left(1+\frac{P_{r}}{\sigma_{n}^{2}}\left|h_{i, j}(\xi)\right|^{2}\right),
$$

where the received power $P_{r}$ corresponding to transmit power $P_{t}$ considering the path loss at distance $d$ and log normal shadowing is given as,

$$
P_{r}=\frac{P_{t}}{d^{2}} \nu,
$$

where $\nu$ is the log-normal shadowing factor i.e. $\log _{10}(\nu) \sim$ $\mathcal{N}\left(0, \sigma_{\nu}^{2}\right)$. Hence, for a given choice of the cluster head, the 
optimal OFDMA subcarrier allocation can be formulated as,

$$
\begin{aligned}
R(\xi) \triangleq \max & \sum_{\substack{i=1 \\
i \neq \xi}}^{N} \sum_{j=1}^{N-1} \rho_{\xi}(i, j) R_{i, j}(\xi) \\
\text { s.t. } \quad \rho_{\xi}(i, j) \in\{0,1\} & \\
& \sum_{\substack{i=1 \\
i \neq \xi}}^{N} \rho_{\xi}(i, j)=1 \\
& \sum_{j=1}^{N-1} \rho_{\xi}(i, j)=1 .
\end{aligned}
$$

Thus, $\rho(i, j) \in\{0,1\}$ is the indicator function denoting the allocation of subcarrier $j$ to cluster member $i, 1 \leq i \leq N-1$ with choice of node $\xi$ as the $\mathrm{CH}$. Hence, the optimal choice of cluster head $\xi^{\star}$ that maximizes the sum data rate can be readily seen to be given as,

$$
\xi^{\star}=\arg \max _{\xi} R(\xi) .
$$

Further, for a scalable video stream, the rate and video quality can be modeled as functions of the quantization parameter $q$ and frame rate $t$ [9] as,

$$
\begin{aligned}
& R(q, t)=\underbrace{R_{\max }\left(\frac{1-e^{-c t / t_{\max }}}{1-e^{-c}}\right)}_{\tilde{R}_{\max }(t)} e^{d\left(1-q / q_{\min }\right)}, \\
& Q(q, t)=Q_{\max }\left(\frac{1-e^{-a t / t_{\max }}}{1-e^{-a}}\right)(\beta q+\gamma),
\end{aligned}
$$

where $R_{\max }, Q_{\max }, a, c, d, \beta$ and $\gamma$ are video sequence characteristic parameters [9]. Hence, for a fixed frame rate $t_{o}$ at rate $R$, the quantization parameter $q$ for the scalable video stream $q$ can be obtained from (4) as,

$$
q(R)=q_{\min }\left\{1-\frac{1}{d} \log \left(\frac{R}{\tilde{R}_{\max }\left(t_{o}\right)}\right)\right\} .
$$

Now substituting the above value of $q(R)$ in (5) yields the video quality $Q(R)$ corresponding to rate $R$ and can be employed to compute the video quality for each cluster member based on the allocation $\rho_{\xi^{\star}}(i, j), 1 \leq i, j \leq(N-1)$. Next we present algorithms for optimal subcarrier allocation $R(\xi), 1 \leq \xi \leq N-1$.

\section{Algorithms FOr WSN Subcarrier Allocation}

For simplicity we assume only one subcarrier per cluster member but the proposed schemes can be extended for multiple subcarriers. The optimal subcarrier allocation problem can be seen to be equivalent to finding a maximum weighted perfect matching in a bipartite graph. Let $G=(V, E)$ denote a graph where $V$ is the set of vertices and $E$ is the set of edges. The quantity $E(i, j)$ represents the edge between vertices $i$ and $j$. The graphs $G$ is bipartite if $V$ can be expressed as $V=A \cup B, A \cap B=\phi$, such that there is no edge between any two vertices belonging to the same set $A$ or $B$. Let $W(i, j)$ denote the weight of the edge $E(i, j)$. Then the maximum weight matching problem is to select a subset of $E$ such the total weight is maximized with the constraint that that no vertex occurs more than once in the mapping, expressed as,

$$
\begin{aligned}
\max & \sum_{i=1}^{|A|} \sum_{i=1}^{|B|} \rho(i, j) W(i, j) \\
& \rho(i, j) \in\{0,1\}, 1 \leq i, j \leq|A| \\
& \sum_{i=1}^{|A|} \rho(i, j)=1 \\
& \sum_{j=1}^{|B|} \rho(i, j)=1,
\end{aligned}
$$

which can be seen to be identical to the rate maximization framework in (2). For each choice of $\mathrm{CH} \xi$ consider $A(\xi)=\mathcal{N} \backslash \xi$, i.e. the set of sensor nodes $\mathcal{N}$ without the $\mathrm{CH} \xi$. Let $B=\mathcal{C}$ where $\mathcal{C}$ denotes the set of subcarriers and $E$ denote set of all possible edges between cluster members and subcarriers, with $E(i, j)$ denoting the edge between the $i^{\text {th }}$ node and $j^{\text {th }}$ subcarrier. Let $W(i, j)=R_{i, j}(\xi)$ denote the weight of edge $E(i, j)$. In the following sections we describe three different matching algorithms, namely Stable matching, Auction based bipartite matching and Hungarian method for intra-cluster sum data rate maximization followed by the optimal $\mathrm{CH}$ selection scheme. We also demonstrate a related scheme to minimize power consumption in fixed data rate based applications. Finally, once $R(\xi)$ is computed for each choice of cluster head $\xi$, the optimal choice of cluster head is given from (3) as the one that maximizes the sum rate for the rate-maximizing subcarrier allocation.

\section{A. Stable Matching using Gale-Shapley Algorithm}

This is a local optimization scheme [10], [11] in which every node is assigned the subcarrier on which it has the highest data rate. In case of conflict, the node with the larger data rate is given higher priority. This scheme yields a sum data rate close to the maximum sum data rate and can be implemented in $\left.O\left(n^{2}\right)\right)$ time. It can be shown that this scheme matches nodes with the channels satisfying the property that for no two pairs $\left(i_{a}, j_{a}\right)$ and $\left(i_{b}, j_{b}\right)$ in the matching $R\left(i_{a}, j_{b}\right)>R\left(i_{a}, j_{a}\right)$ and $R\left(i_{b}, j_{a}\right)>R\left(i_{b}, j_{b}\right)$. Hence, this scheme is also termed as stable matching. There exist multiple matchings satisfying the stability criterion, while the above scheme determines the matching having the highest sum data rate amongst all stable matchings. The scheme is described in Algorithm 1 in which the function $i s \_t h e r e \_a \_z e r o(\mathbf{A})$ checks if any entry of matrix $\mathbf{A}$ is 0 . The function indices_sort $(A)$ returns an array containing indices of elements corresponding to input array $A$ sorted in descending order.

\section{B. Multi-Item Auction based Bipartite Matching}

The multi-item auction problem [12], [13] in game theory considers the following allocation scenario. There are $n$ buyers, $m$ items and each buyer can buy at most one item. The 


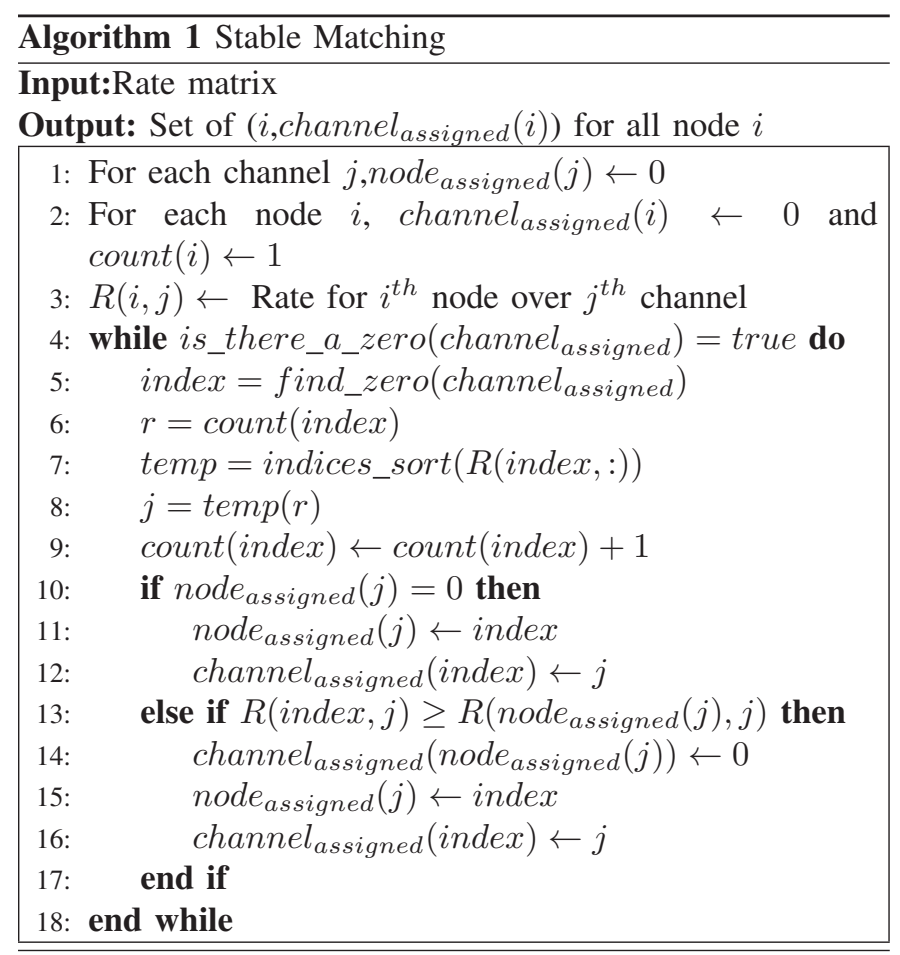

aim of the auction is to maximize the total profit i.e. the sum of profits of all the buyers and sellers. This is thus equivalent to the maximum weighted bipartite matching with the bidders and items being the vertices of the bipartite graph and the profit corresponding to the $i^{\text {th }}$ buyer buying the $j^{\text {th }}$ good denoting the weight $W(i, j)$ of the corresponding edge. An ascending price simultaneous auction can be employed to maximize the overall profit or equivalently the sum rate in this case. The resultant sum data rate is within $N \delta$ of the maximum sum data rate where $N$ is the number of nodes and $\delta$ is a parameter employed in the algorithm. Hence, selecting a small value for $\delta$ yields the maximum sum data rate but might require more iterative steps to compute the optimal allocation as the time complexity is $O\left(n^{2} / \delta\right)$. But it generally converges faster i.e takes much less number of steps than $\left(n^{2} / \delta\right)$ to give the solution. The steps of the procedure are described in Algorithm 2. The advantage of this algorithm is that being distributed in nature, it can be implemented in a decentralized fashion.

\section{Hungarian Algorithm for Maximal Matching}

This algorithm is based on Kuhn-Munkres theorem and yields the maximum sum data rate in $O\left(n^{3}\right)$ time [14]. It converts the weighted bipartite matching problem to an unweighted bipartite matching problem which can be easily solved using the maxflow algorithm [14]. This is described in Algorithm 3 . It converts the weighted bipartite graph having vertices $(\mathcal{N}, \mathcal{C})$ with Rate $(\mathbf{R})$ as the associated edge weight matrix to an unweighted bipartite graph with same vertices and set of edges $E_{l}$, in which any perfect matching [14] is the required solution. In the pseudo code the function $C O N$ NECTED checks the connectedness of a graph [15], PERFECT checks if all vertices (nodes or channels) are connected in the

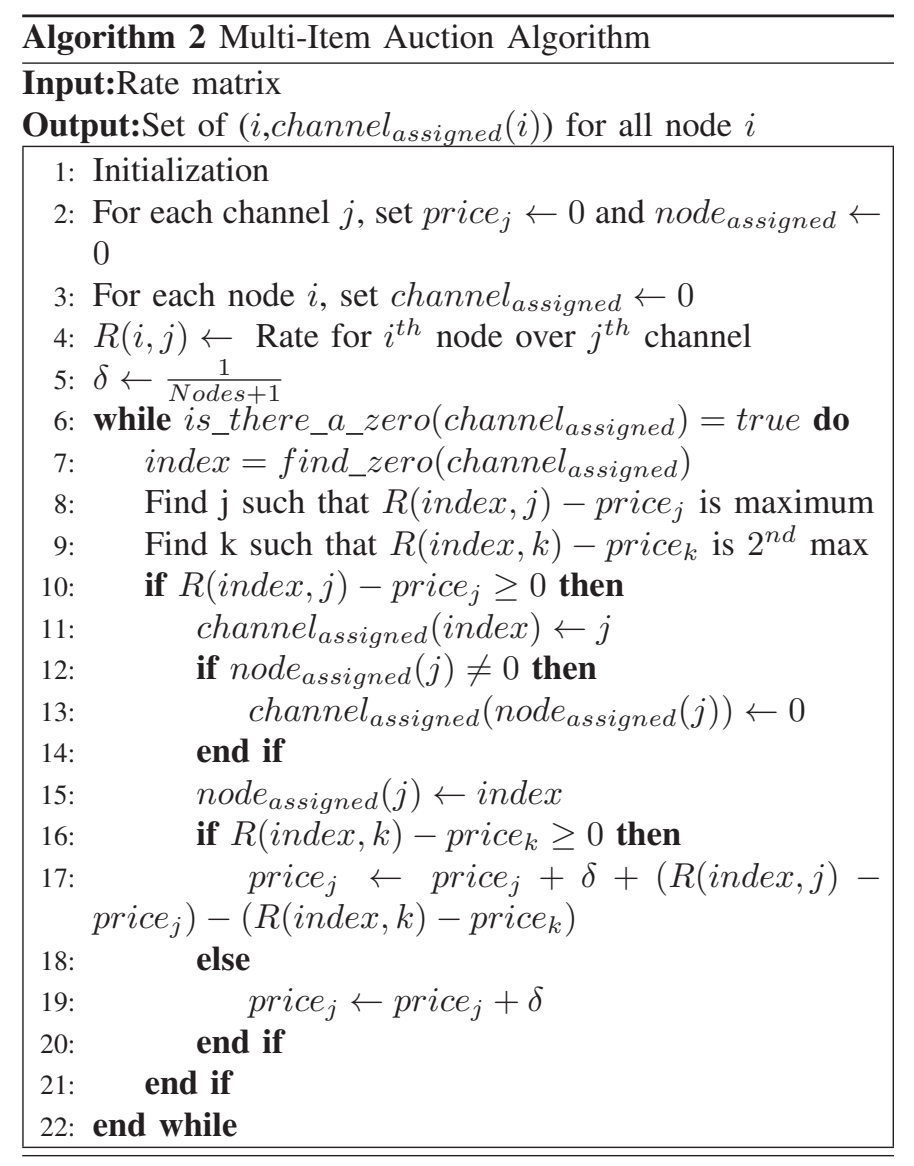

matching $\mathcal{A}$ and find_perfectMatch returns a perfect matching $\mathcal{A}$ of the graph.

\section{Power minimization and Sum Video Quality maximization} schemes

Since sum video quality maximization problem is similar to sum data rate maximization, the matching algorithms described above can be used to find the optimal subcarrier allocation scheme for video quality maximization simply by employing the quality matrix $\mathbf{Q}(\xi)$ with each entry $Q^{(\xi)}(i, j)$ denoting the video quality attainable on subcarrier $j$ by node $i$, with choice $\xi$ for the cluster head. Each quality $Q^{(\xi)}(i, j)$ can be derived from the corresponding $R^{(\xi)}(i, j)$ as described in (5) in section [II. Further, if $R^{(\xi)}(i, j)>R_{\max }$ of the corresponding video sequence, then $Q^{(\xi)}(i, j)$ is maximum i.e equal to $Q_{\max }$. In this case the transmit power can be decreased such that $R^{(\xi)}(i, j)=R_{\max }$ without affecting the overall video quality.

Similarly, the power minimization problem can be formulated as a maximization problem and the above algorithms can be employed to find the desired solution. Let $R$ denote the required data rate. Employing (1), the required transmit power $P^{(\xi)}(i, j)$ can be computed for each node $i$ on each subcarrier $j$. Let $P_{\max }^{(\xi)}$ denote the maximum value in the power matrix $\mathbf{P}^{(\xi)}$. One can now construct the weight matrix $\mathbf{W}^{(\xi)}$ by subtracting each element of matrix $\mathbf{P}^{(\xi)}$ from $P_{\text {max }}^{(\xi)}$. By construction, the maximum weighted matching in $\mathbf{W}^{(\xi)}$ yields 


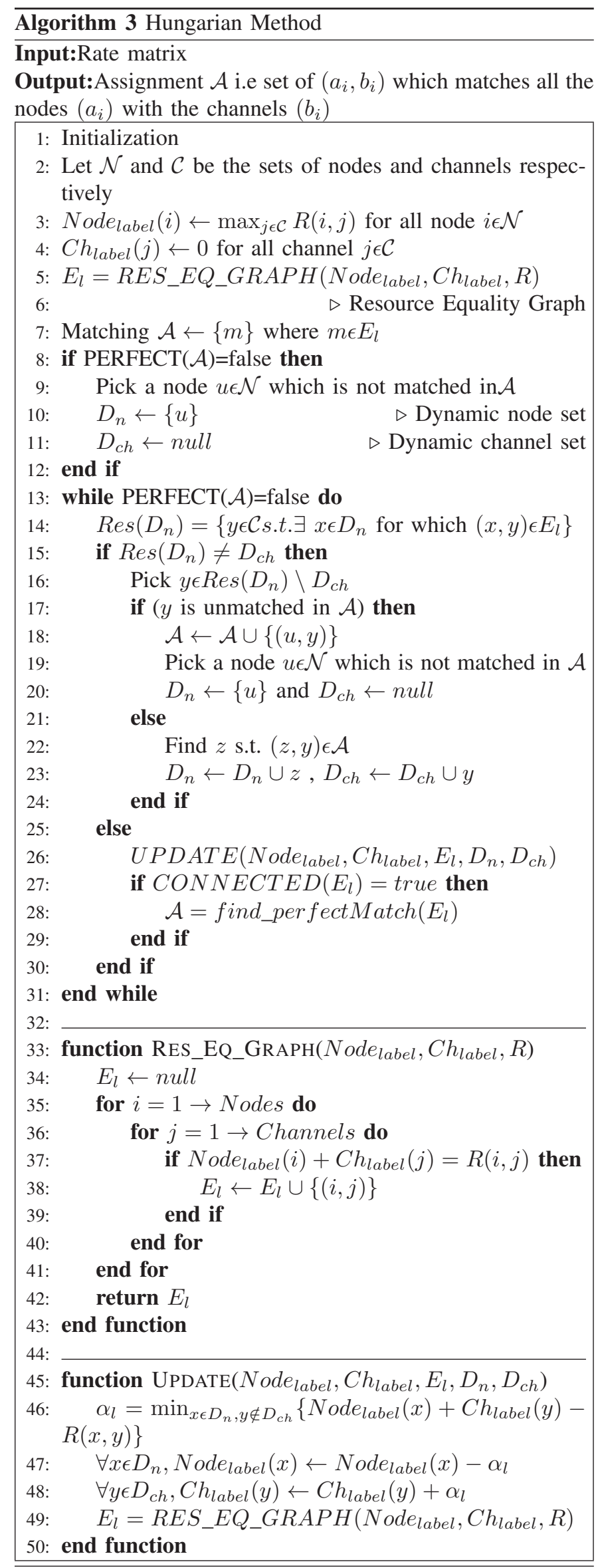

the minimum power matching in $P_{\max }^{(\xi)}$. Finally, choosing the cluster head $\xi$, one can compute the optimal $\mathrm{CH}$ assignment and subcarrier allocation for total power minimization.

\section{SIMULATION RESULTS}

We implemented our simulation setup in MATLAB R2011b. In our WSN sum data rate simulation we consider a transmit power of $P_{t}=20 \mathrm{~dB}$ per sensor node and a bandwidth of $B=262.56 \mathrm{KHz}$ per subcarrier. We compute the sum data rate obtained from the subcarrier allocation and $\mathrm{CH}$ assignment schemes described in section [II] Fig 3 demonstrates that the stable matching for $\mathrm{CH}$ assignment and subcarrier allocation in section III-A leads to a considerable increase in the sum data rate over a random subcarrier allocation scheme. Further, this difference in sum data rate increases with the cluster size of the WSN. It also demonstrates that the stable matching and auction based bipartite matching scheme produce similar sum rate with the multi-item auction based scheme slightly outperforming stable matching. Hungarian method and auction based matching give same results.

For video quality simulation, we have employed the Akiyo CIF video sequence, with $Q_{\max }=1, P_{t}=20 \mathrm{~dB}$ transmit power for each sensor node and a bandwidth of $B=262.56$ $\mathrm{KHz}$ per subcarrier. Fig 4 shows the sum video quality for all the competing $\mathrm{CH}$ selection schemes for different number of nodes in the WSN cluster. As expected, one can see a trend similar to that for sum data rate. The sum video quality can be seen to be maximum for the Hungarian method and auction based matching and slightly lower for Stable allocation. These three schemes vastly outperform the random allocation scheme with the difference in sum video quality increasing with the number of nodes in the cluster. Finally Fig 5 shows the relative frequency of being chosen as the $\mathrm{CH}$ for uniformly distributed nodes in the cluster. As can be seen, all the nodes have a similar probability of $\mathrm{CH}$ role assignment. Hence, the average power consumption is uniform across cluster members, thus maximizing the WSN life.

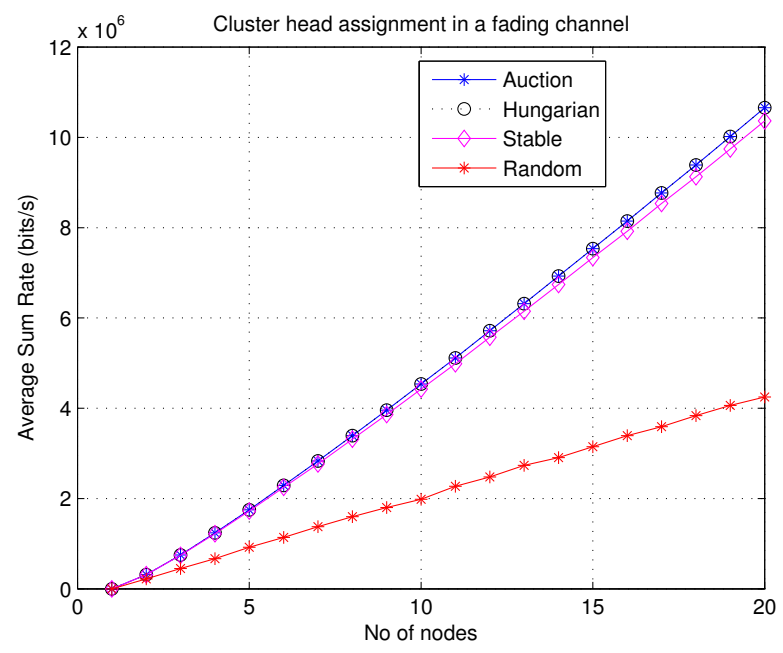

Figure 3: Sum Rate Comparison 


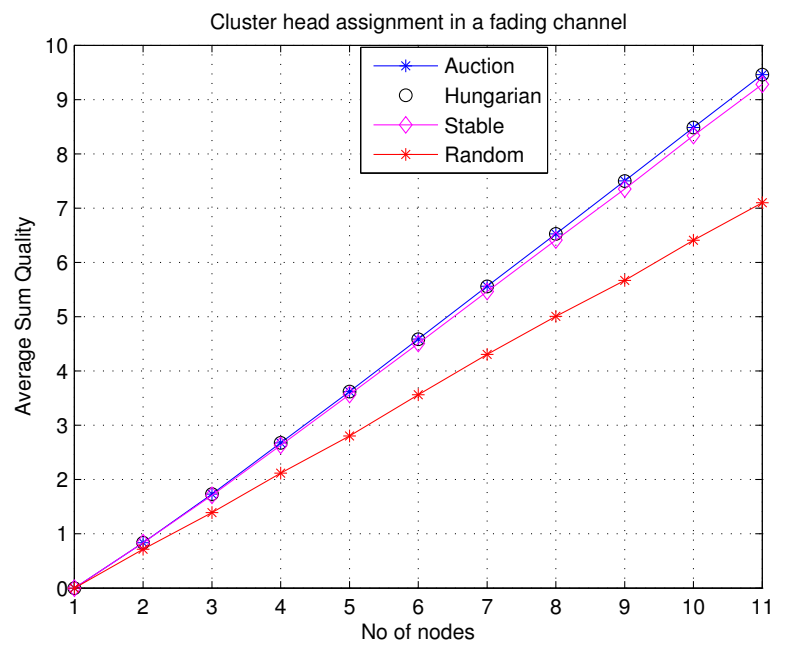

Figure 4: Sum video Quality Comparison

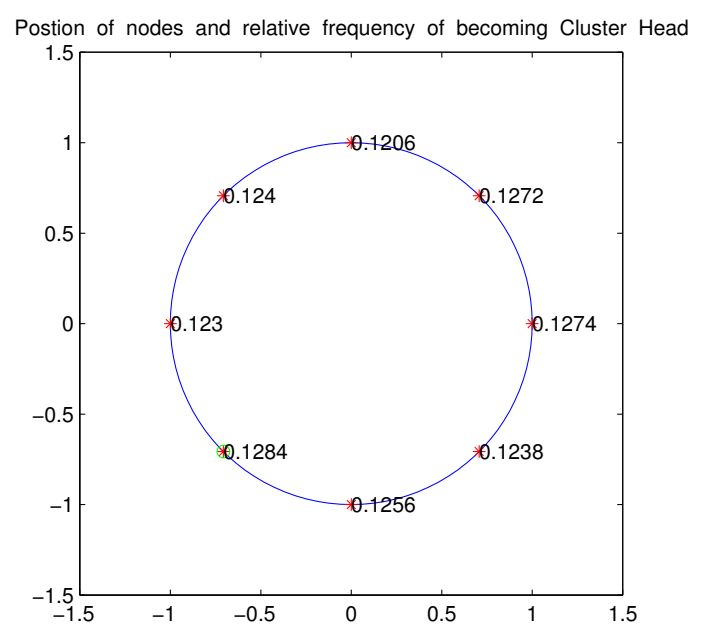

Figure 5: Load distribution

\section{Conclusion}

In this work we described three optimal bipartite matching based schemes for $\mathrm{CH}$ selection and subcarrier allocation among the cluster nodes for sum data rate maximization in a OFDMA based hierarchical WSN. The different schemes are of varying complexity and performance. While stable matching computes the optimal assignment close to the maximum value with $O\left(n^{2}\right)$ ) complexity, the Hungarian method finds the maximum weighted matching in $O\left(n^{3}\right)$ time. The MultiItem auction based matching is adaptive as its execution time and performance depend on user specified parameter $\delta$, with its time complexity $O\left(n^{2} / \delta\right)$. Further, the sum rate is within $n \delta$ range of maximum value. Hence, depending upon the specific requirement, a matching scheme can be selected suitably. However it should be noted that the worst case time complexity analysis does not always give a good estimate especially in case of Auction algorithm which generally converges much faster than $O\left(n^{2} / \delta\right)$. Further, we demonstrated that these algorithms can also be applied to maximize the sum video quality or minimize the total power dissipation of the network which increases network life. In a conventional TDMA based WSN the rate and thus video quality are compromised to achieve uniformity in energy consumption among the nodes. We also illustrated that for uniformly distributed nodes in the cluster and random fading channels, these schemes result in equal average energy consumption among the nodes without any compromise in the sum data rate or video quality. These scheme can also be extended for the general case involving multiple subcarriers per node.

\section{REFERENCES}

[1] I. F. Akyildiz and M. C. Vuran, Wireless Sensor Networks. Wiley, Aug. 2010.

[2] A. A. Abbasi and M. Younis, "A survey on clustering algorithms for wireless sensor networks," Comput. Commun., vol. 30, no. 14-15, pp. 2826-2841, Oct. 2007. [Online]. Available: http://dx.doi.org/10.1016/j.comcom.2007.05.024

[3] S. Chatterjee and M. Singh, "A centralized energy-efficient routing protocol for wireless sensor networks," Int. J. Advanced Networking and Applications Volume, vol. 03, no. 5, pp. 12-18, 2012.

[4] Y. J. Zhang and K. Letaief, "Multiuser adaptive subcarrier-and-bit allocation with adaptive cell selection for OFDM systems," Wireless Communications, IEEE Transactions on, vol. 3, no. 5, pp. 1566-1575, Sept.

[5] Y. Wang, J. Ostermann, and Y.-Q. Zhang, Video Processing and Communications. Prentice Hall, Aug. 2002.

[6] I. F. Akyildiz, T. Melodia, and K. R. Chowdhury, "A survey on wireless multimedia sensor networks," Comput. Netw., vol. 51, no. 4, pp. 921-960, Mar. 2007. [Online]. Available: http://dx.doi.org/10.1016/j.comnet.2006.10.002

[7] E. Gürses and O. B. Akan, "Multimedia communication in wireless sensor networks," Annales des Télécommunications, vol. 60, no. 7-8, pp. 872-900, 2005.

[8] S. Misra, M. Reisslein, and G. Xue, "A survey of multimedia streaming in wireless sensor networks," Communications Surveys Tutorials, IEEE, vol. 10, no. 4, pp. 18-39, Quarter.

[9] S. Parakh and A. K. Jagannatham, "Optimal resource allocation and VCG auction-based pricing for H.264 scalable video quality maximization in 4G wireless systems," Advances in Multimedia, vol. 2012, 2012.

[10] http://www.cse.iitd.ernet.in/rahul/cs905/lecture16/index.html

[11] D. Gale and L. S. Shapley, "Least squares quantization in PCM," The American Mathematical Monthly, vol. 69, no. 1, pp. 9-15, jan 1962.

[12] http://agtb.wordpress.com/2009/07/13/auction-algorithm-for-bipartite-matching

[13] http://www.cse.iitd.ernet.in/rahul/cs905/lecture17/index.html

[14] http://www.cs.ucsb.edu/suri/cs231/Matching.pdf

[15] http://en.wikipedia.org/wiki/Bipartitegraph 